\title{
Ethylene is Involved in the Actin Cytoskeleton Rearrangement During the Root Gravitropic Response of Arabidopsis thaliana ${ }^{1}$
}

\author{
G. A. Pozhvanov ${ }^{a}$, A. E. Gobova ${ }^{a}$, M. P. Bankin ${ }^{a}$, K. Vissenberg ${ }^{b, c}$, and S. S. Medvedev ${ }^{a}$ \\ ${ }^{a}$ Faculty of Biology, St. Petersburg State University, Universitetskaya nab. 7-9, St. Petersburg, 199034 Russia \\ ${ }^{b}$ Biology Deptartment, University of Antwerp, Prinsstraat 13, Antwerp, 2000 Belgium \\ ${ }^{c}$ Plant Biochemistry and Biotechnology Laboratory (PBBL), Department of Agriculture, School of Agriculture, \\ Food and Nutrition, Technological and Educational Institute of Crete, Heraklion, PC 71410 Greece \\ e-mail:g.pozhvanov@spbu.ru \\ Received January 20, 2016
}

\begin{abstract}
Gravitropism, the directed plant growth with respect to the gravity vector, is regulated by auxin and its polar transport system, several secondary messengers, and by the cytoskeleton. Recently we have shown that the actin cytoskeleton in the root transition zone of Arabidopsis thaliana (L.) Heynh was rearranged after gravistimulation (rotation by $90^{\circ}$ ): the fraction of axially aligned microfilaments decreased and the fraction of oblique and transversally-oriented microfilaments increased. In the present research we have studied the effect of ethylene and inhibitors of its synthesis on actin cytoskeleton rearrangement during the gravitropic response. Application of the ethylene releasing substance ethephon to A. thaliana seedlings led to the disassembly of actin microfilaments as well as their broad angle distribution in cells of the root transition zone. This actin rearrangement was escaped by treatment with the ethylene synthesis inhibitor aminoethoxyvinylglycine (AVG). Another negative regulator of ethylene, salicylic acid, was shown to disturb actin microfilament rearrangement as well. We conclude that ethylene is essential for the process of actin cytoskeleton rearrangement in root cortex cells during the gravitropic bending response.
\end{abstract}

Keywords: Arabidopsis thaliana, gravitropism, ethylene, cytoskeleton, actin, root, elongation zone, ethephon, salicilyc acid, aminoethoxyvinylglycine

DOI: $10.1134 /$ S1021443716050095

\section{INTRODUCTION}

Gravitropism is the key response of plants to the gravity force. Gravitropism is referred to as the directed growth of plant organs with respect to the gravity vector. The most widely known demonstrations of gravitropism are the upward growth of shoots against the gravity direction (negative gravitropism) and the downward growth of roots co-directional with the gravity vector (positive gravitropism). The plant organ gravitropic bending in response to a change of its position in space is another example of gravitropism. Plants are able to respond to an extremely weak gravitational impact. Several events are detected at early stages of the plant gravitropic response: electrical polarization, formation of polar fluxes and gradients of auxin and calcium, which together lead to establishment of a new physiological polarity axis within $10 \min [1,2]$.

Ethylene is another important element in the regulation of the plant gravitropic response. Its effects on

\footnotetext{
${ }^{1}$ The article was translated by the authors.

Abbreviations: AVG-L- $\alpha-(2-$ Aminoethoxyvinyl)glycine; ACC1 -aminocyclopropane-1-carboxylic acid; fABD2-second actinbinding domain of fimbrin 1; GFP-green fluorescent protein.
}

growth processes were first described by Dimitry Neljubow [3]. By that time it was well known that illumination of laboratory plants with so-called "lighting gas" caused growth anomalies: growth of shoots was inhibited, their thickness was increased, and their growth turned to horizontal. Neljubow showed that laboratory plant shoots switched to horizontal growth only after the addition of the "lighting gas" to the fresh air. Among all components of the "lighting gas" obtained by oil pyrolysis-hydrogen sulfide, acethylene, benzene, xylol, naphthalene and ethyleneonly acethylene and ethylene in very low concentrations caused the so-called "triple response"-i.e. horizontal growth, inhibition of shoot elongation and shoot lateral enlargement.

On one hand, D. Neljubow was the first to establish that plant growth abnormalities in laboratory conditions were caused by the presence of ethylene in the "lighting gas". On the other hand, he discovered a new phenomenon in plant physiology, namely the switch of shoot growth direction to the horizontal under the treatment with ethylene.

The actin cytoskeleton plays an important role in gravitropism as well. However, its functions differ at 
subsequent stages of the gravitropic response. Microfilaments act as a negative regulator of gravitropism at the gravity perception stage by preventing too fast statolith sedimentation in the direction of the gravity force $[4,5]$. Later, during the gravity signal transduction stage and the growth bending response, the actin cytoskeleton turns into a positive regulator of gravitropism. It is required for vesicular transport resulting in polar distribution of auxin carriers in the plasma membrane [6] and it is necessary for modulating cell extension growth at both sides of the organ [7], by which the gravitropic bending is being developed. Nevertheless, the mechanism of regulation of actin rearrangement during gravitropism is still unclear.

We have shown earlier that Arabidopsis seedling reorientation with respect to the gravity vector triggered actin microfilament rearrangement in the root transition zone [8]. The fraction of axially aligned microfilaments decreased while the fraction of oblique and transversally oriented microfilaments increased. It is known that in plants the organisation of the actin cytoskeleton could be influenced by hormonal status $[9,10]$. However, the mechanism of interaction between hormone dynamics and cytoskeleton organisation during plant gravitropic responses is not yet sufficiently understood [5]. This study is focused on the analysis of effects of ethylene and inhibitors of its synthesis on actin microfilament rearrangement during the Arabidopsis root gravitropic response.

\section{MATERIALS AND METHODS}

Plant material and growth conditions. Experiments were performed with 6-day-old seedlings of Arabidopsis thaliana (L.) Heynh, ecotype Columbia 0, transformed with the GFP- $F A B D 2$ construct [11], if not otherwise indicated. GFP-fABD2 plants constitutively express GFP fused with a second (C-terminal) actin-binding domain of fimbrin 1 (fABD2), allowing in vivo visualization of the actin cytoskeleton. Plants were grown in sterile conditions on the surface of solidified half-strength Murashige-Skoog nutrient medium (MS/2, Duchefa, the Netherlands) containing $1 \%$ (mass/vol.) sucrose (Ecros, Russia) and $0.35 \%$ (mass/vol.) phytagel (Sigma-Aldrich, United States) in vertically-oriented square Petri plates $120 \times$ $120 \times 17 \mathrm{~mm}$ (Greiner Bio-One, Germany). Seeds were stratified for one day at $4^{\circ} \mathrm{C}$, then moved to a MLR-351 climate chamber (Panasonic, Japan) and grown at the light intensity of $5000 \mathrm{~lx}$ provided by FL40SS-W/37 fluorescent lamps, in a 16-hour photoperiod at $20^{\circ} \mathrm{C}$.

Gravistimulation and treatment of plants. Seedlings were transferred to a vertically placed objective slide, covered with a coverslip $(24 \times 50 \mathrm{~mm}$, Thermo Scientific, Germany) and treated for 30-60 min with $100 \mu \mathrm{L}$ of $10 \mu \mathrm{M}$ salicylic acid, $10 \mu \mathrm{M}$ aminoethoxyvinylglycine (AVG) or $10 \mu \mathrm{M} 2$-chloroethylphosphonic acid (ethephon) solution in Tris/Mes buffer ( $2 \mathrm{mM} / 4 \mathrm{mM}), \mathrm{pH}$ 6.0. Control plants were treated with the same buffer only. Solutions of salicylic acid and AVG were prepared from $5 \mathrm{mM}$ stock in water : DMSO mixture $(1: 1)$, stored at $-20^{\circ} \mathrm{C}$. The solution of ethephon was freshly prepared before every experiment. All chemicals were from Sigma-Aldrich (United States).

Gravistimulation was performed by a $90^{\circ}$ rotation of objective slides with plants in the vertical plane.

Actin cytoskeleton visualization was performed using a Leica TCS SP5 inverted confocal laser scanning microscope (Germany), equipped with a $40 \times$ objective lens with numerical aperture of 1.3 and oil immersion. Fluorescence was induced with a $488 \mathrm{~nm}$ argon laser (20-35\% power) and emission was recorded in the range of 505-585 nm (gain 80). Optical sections $(280 \times$ $280 \mu \mathrm{m}$ ) were made at $2.48 \mu \mathrm{m}$ intervals.

Confocal microscopy experiments were carried out at Research Resource Center "Molecular and Cell Technologies" of St. Petersburg State University.

Analysis of actin cytoskeleton organization. Maximal projections of $8-10$ optical sections through the stele and root cortex in the transition zone were made with Leica Aplication Suite 2010. Actin microfilament detection and their angle distribution were performed automatically using the Microfilament Analyzer software [12] with the following settings: contrast 1.5, $\mathrm{min}$ length $10 \mathrm{px}$, diameter $1 \mathrm{px}$, detection threshold $3 \times$, angular step $3^{\circ}$.

Five different cells from the root transition zone were studied in at least 20 different roots per experimental treatment. Automatically detected microfilaments were classified as axial, oblique or transverse, if they formed an angle of, respectively, $0-30^{\circ}, 30-60^{\circ}$ or $60-90^{\circ}$ with the root axis, as shown on Fig. 1. As a result, axially oriented microfilaments were aligned parallel to the root apical-basal axis [13], and transversally oriented microfilaments were perpendicular to the root axis. In case of vertical growth, axial microfilaments were co-directed with the gravity vector, and transversal ones were perpendicular to it. In contrast, after gravistimulation of plants axial microfilaments became perpendicular to the gravity vector, and transversal ones became aligned with the gravity direction. Data on histograms show the abundance (the fraction in \%) of axial, oblique and transversally oriented microfilaments in confocal images.

Analysis of root growth rates. The root growth rate of Arabidopsis seedlings was measured using a motorized imaging setup, consisting of a Canon EOS 7D camera equipped with EF-S $60 \mathrm{~mm} \mathrm{f} / 2.8$ Macro USM lens (Canon, Japan) that was moved with precision by robotized rails (Zaber, Canada) in front of a row of Petri plates with $A$. thaliana seedlings at the laboratory of Prof. K. Vissenberg (University of Antwerp). Root length was measured from acquired serial images in Image J (NIH, United States).

Statistical data analysis. All experiments were performed in at least three replicates. Statistical data anal- 
ysis was performed in Microsoft Excel and significance was estimated using Student's $t$-test. Bars on histograms (Figs. 4 and 5) show the standard error.

\section{RESULTS AND DISCUSSION}

The actin cytoskeleton was revealed in all cell types of Arabidopsis thaliana GFP- $f A B D 2$ roots from the root apical meristem to the elongation zone. Figures $2 \mathrm{a}, 2 \mathrm{e}$ and $2 \mathrm{i}$ show confocal images of the actin cytoskeleton in the root transition zone of vertically oriented seedlings observed for 30-60 min. In stele cells actin microfilaments were organized into thick bundles with predominantly axial or oblique orientations. In the root cortex and rhizoderm the actin cytoskeleton was organized into thinner filaments which were parallel to the root axis or even oblique. Filaments directed to lateral plasma membrane were minor. Analysis of actin microfilament angle distribution in the transition zone (Figs. 3a, 3b and 3f) showed that the angle distribution spectrum practically did not change within 30-60 min of observation. More than $50 \%$ of the microfilaments were oriented along the root axis (Fig. 4).

After a $90^{\circ}$-rotation of plants in the vertical plane (gravistimulation), we have found a number of changes in the organisation of actin in the root transition zone after 15 min of observation (Figs. 2m, 2q and $2 \mathrm{u}$ ). The fraction of axially aligned microfilaments decreased with subsequent increase of fractions of oblique and transversally oriented microfilaments (see circular diagrams on Fig. $3 \mathrm{j}$ ). The same trend persisted after 30 and $60 \mathrm{~min}$ of gravistimulation (Figs. 3k and 3o): the microfilament angle distribution spectrum became more broad with predominant oblique microfilaments (Fig. 4). These data confirm and clarify what we have found previously for actin cytoskeleton rearrangement during a gravity response [8]. To conclude, the gravitropic response of the $A$. thaliana root transition zone is featured by the assembly of actin microfilaments parallel to the gravity vector.

\section{Ethylene Promotes Actin Cytoskeleton Disassembly and Broadens the Spectrum of Actin Microfilament Angle Distribution in the Arabidopsis Root Transition Zone}

Disturbance of the vertical orientation of axial organs appears to be a stressful situation for a plant. Apparently because of that, biosynthesis of the stress hormone ethylene is activated during plant gravitropic responses, and inhibitors of ethylene synthesis and action suppress the gravitropic response [14-16]. On the other hand, there are data that do not support the involvement of ethylene in the primary gravitropic response [17], so it is still the question what the exact role of ethylene is in gravitropic responses.

At the time, Neljubow [3] payed his attention to the fact that ethylene treatment affected the "geotropism properties" of plants and caused the switch of stem growth orientation from vertical to horizontal, i.e. eth-

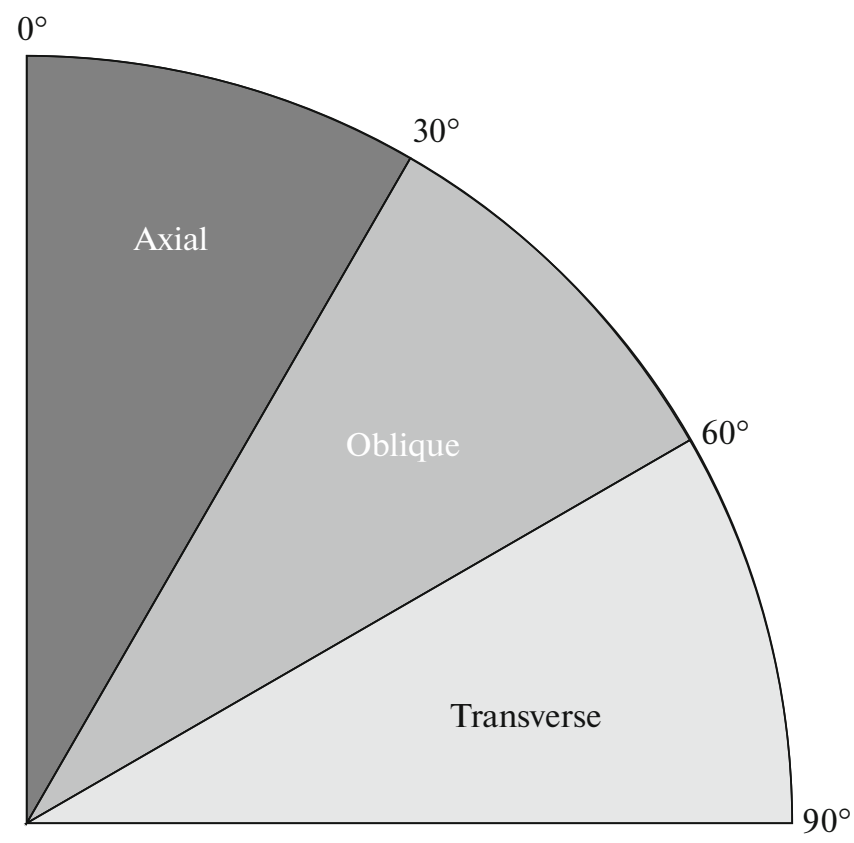

Fig. 1. Microfilament classification based on their orientation with respect to the root axis of $A$. thaliana. Axially oriented microfilaments: $0-30^{\circ}$, oblique: $30-60^{\circ}$, transverse: $60-90^{\circ}$.

ylene is involved into plant gravitropic responses by triggering the plagiotropic growth of shoots.

Ethephon (2-chloroethylphosphonic acid) is commonly used in physiological experiments as the ethylene producer, it is well soluble in water at $\mathrm{pH}>4.5$ and is easily decayed releasing ethylene.

In our experiments, treatment with ethylene of vertically growing $A$. thaliana seedlings caused a partial disassembly of the actin cytoskeleton in the root transition zone (Figs. 2f and 2j). Small fluorescent aggregates (dots) appeared in the root cortex and in stele, whereas longer filaments with lengths comparable to the diameter of the cell disappeared after $30 \mathrm{~min}$ of treatment (Fig. 2f). In addition, the spectrum of actin microfilament orientation became dramatically broad after 30 min of treatment with ethylene, which was characteristic for actin rearrangement in gravistimulated Arabidopsis roots (Figs. 3c and 3g). Among microfilaments, the oblique orientation was predominant (Fig. 5a). Despite that, after 60 min of treatment with the ethylene producer, axially aligned microfilaments were most abundant in the root cortex again (Fig. 5a), like in the vertically-grown control, but the total number of microfilaments continued to decrease (Figs. 3c and 3g).

In contrast, treatment with ethephon during gravistimulation had the opposite effect: actin microfilament angle distribution after $30 \mathrm{~min}$ of gravistimulation did not differ from the vertically-grown control (Fig. 5b). Moreover, the fraction of axially aligned microfilaments increased slightly, and the angle distri- 
Time, $\min$
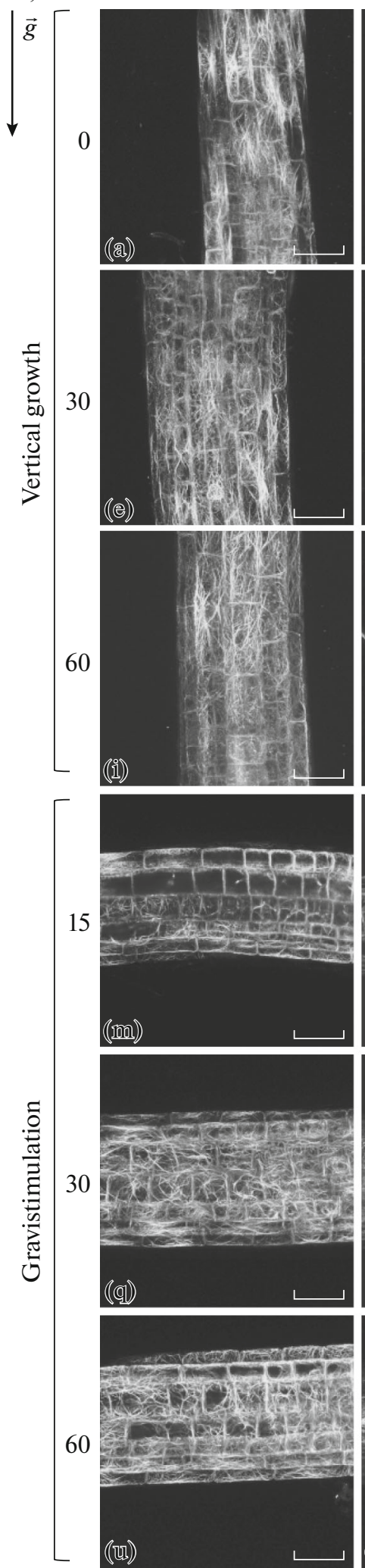

Ethephon
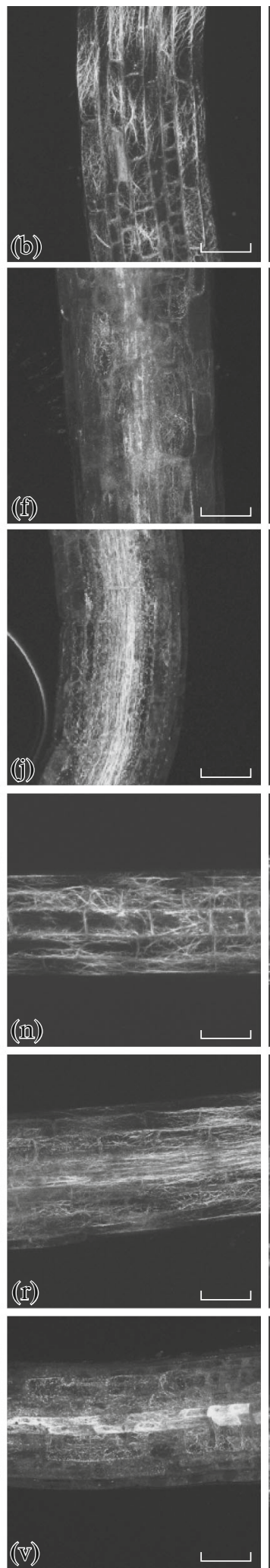

SA
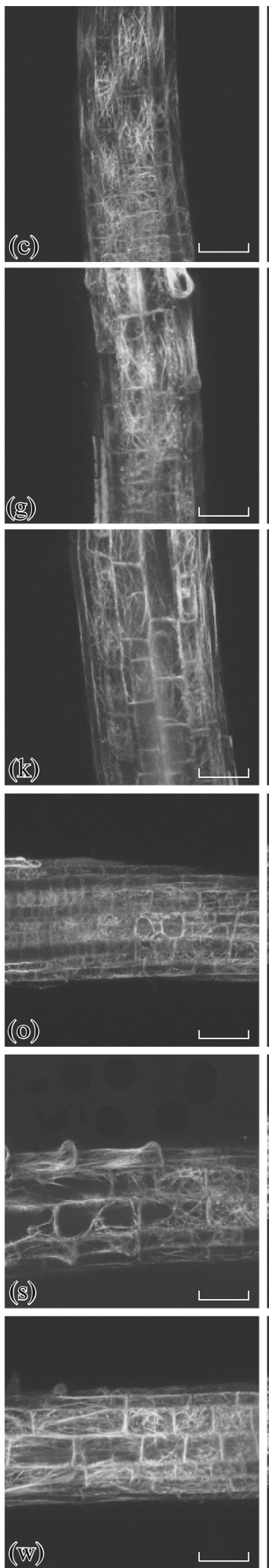

AVG
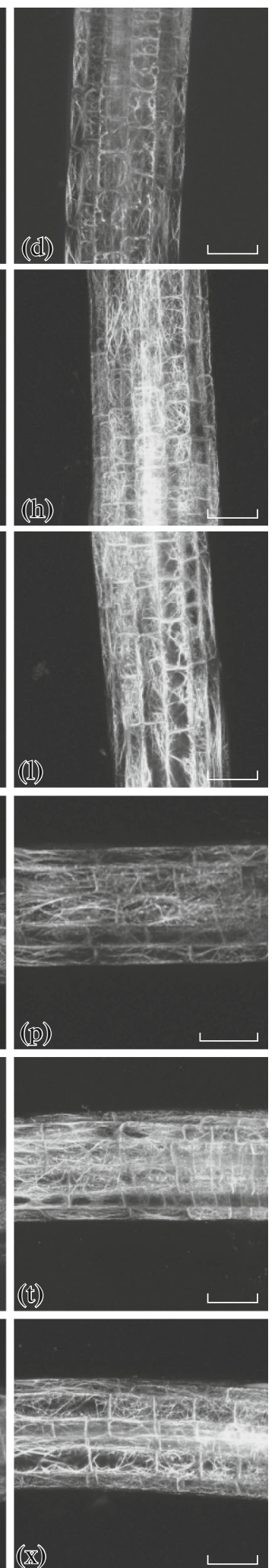

Fig. 2. Actin cytoskeleton organization in the distal elongation zone of $A$. thaliana $G F P-f A B D 2$ roots. The actin cytoskeleton was visualized in vivo in the distal elongation zone of 6 -day-old seedling roots under vertical growth conditions $(\mathrm{a}-1)$ or after gravistimulation $(\mathrm{m}-\mathrm{x})$ by $90^{\circ}$ rotation in the vertical plane during 0-60 min treatment with Tris/Mes buffer $(2 \mathrm{mM} / 4 \mathrm{mM}$, pH 6.0) containing $10 \mu \mathrm{M}$ ethephon, salicylic acid (SA) or aminoethoxyvinylglycine (AVG). Maximal projections of 8-10 confocal optical sections through the stele and cortex in the root elongation zone are shown. Either figure demonstrates a typical image from corresponding experimental series (at least 20 roots for either experiment). Scale bar: $50 \mu \mathrm{m}$. 


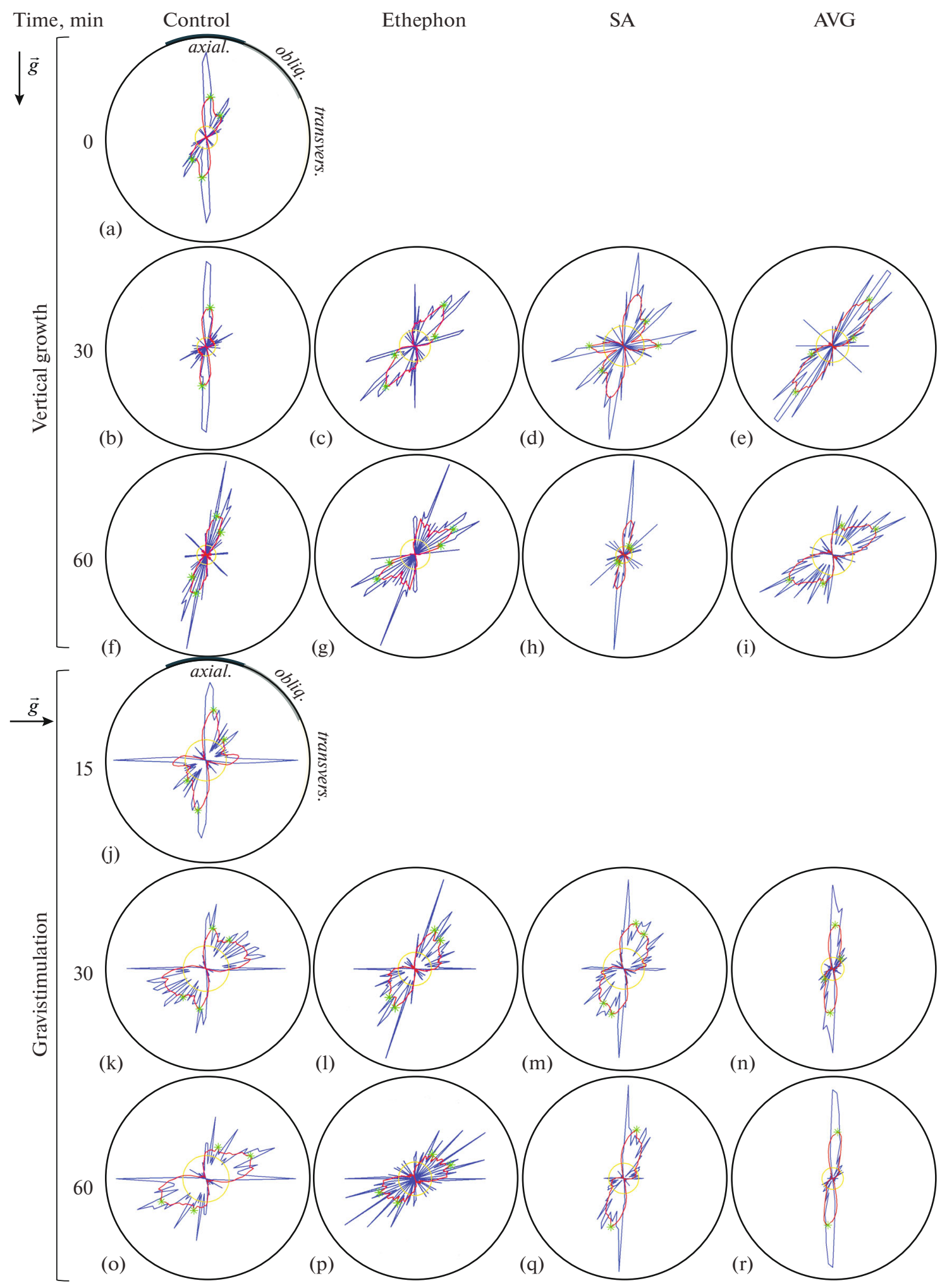

Fig. 3. Angle distribution of actin microfilaments in distal elongation zone of $A$. thaliana $G F P-f A B D 2$ roots. Microfilament angle distribution was analyzed in cortical cells of the distal elongation zone in 6-day-old roots under vertical growth conditions (a-i) or after gravistimulation ( $\mathrm{j}-\mathrm{r})$ during $0-60 \mathrm{~min}$ treatment with Tris/Mes buffer $(2 \mathrm{mM} / 4 \mathrm{mM}, \mathrm{pH}$ 6.0) containing $10 \mu \mathrm{M}$ ethephon, salicylic acid (SA) or aminoethoxyvinylglycine (AVG). Analyzis of maximal projections of 8-10 confocal optical sections was performed in Microfilament Analyzer [12]. Microfilaments were classified as axially oriented, if their inclination to the root axis was $0-30^{\circ}$, oblique: $30-60^{\circ}$, and transversally oriented: $60-90^{\circ}$. Typical diagrams are shown (at least 20 roots for either experiment). 

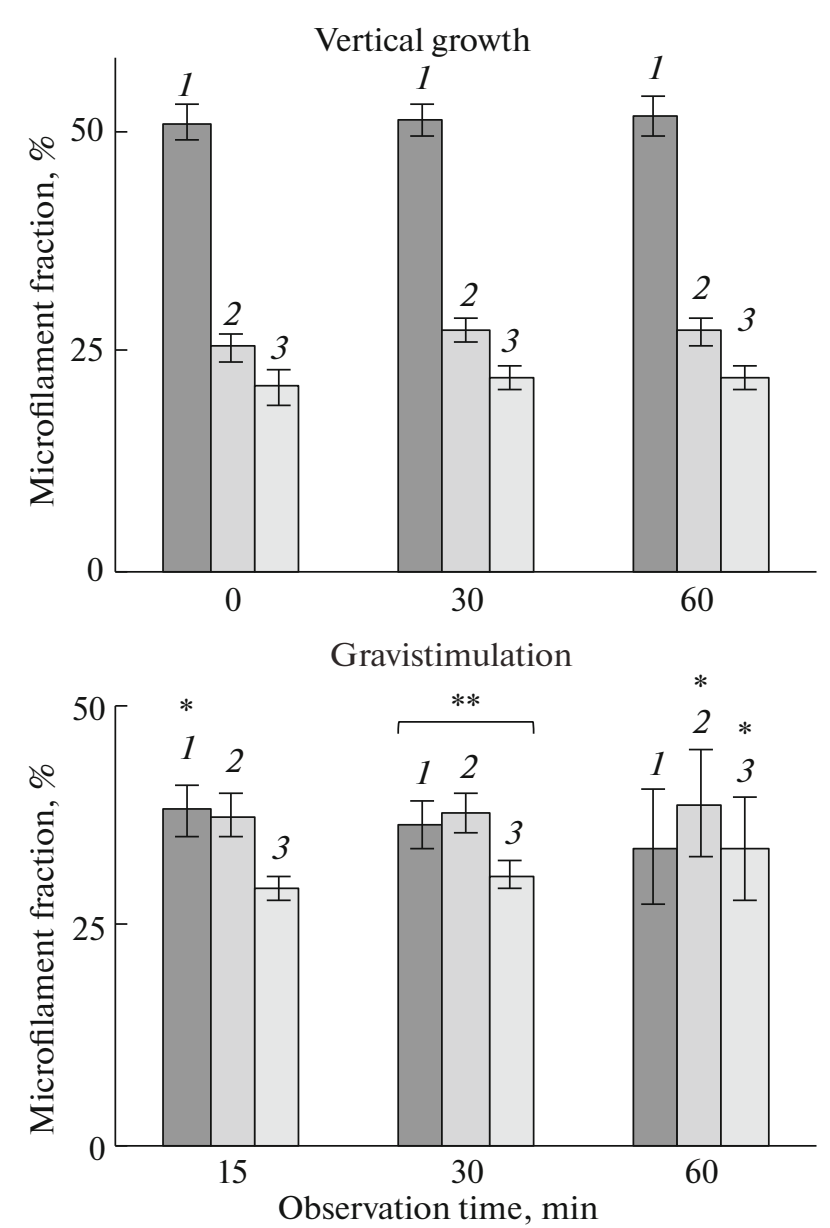

Fig. 4. Classes of microfilaments oriented axially (1), obliquely (2) or transversally (3) with respect to the root apical-basal axis in cortical cells of the distal elongation zone of 6-day-old $A$. thaliana $G F P-f A B D 2$ roots, grown in vertical position or after gravistimulation for $15-60 \mathrm{~min}$. Asterisks show significance of differences with the level of vertically oriented plants (Student's $t$-test): ${ }^{*} 0.01<P<$ 0.05 ; ** $P<0.01$. Bars show the standard error.

bution spectrum was narrower than in gravistimulated plants but not treated with ethephon (Figs. 3k and 31). Exogenous ethylene presumably inhibited actin cytoskeleton rearrangements triggered by gravistimulation. The fraction of oblique microfilaments increased (Fig. 5b) and the fraction of transversally oriented microfilaments decreased by $60 \mathrm{~min}$ of gravistimulation with ethylene treatment. The ratio between three classes of microfilament orientation (Figs. 31 and 3p) became the same as in control roots after $30 \mathrm{~min}$ of gravistimulation (Fig. 4). In contrast to the vertically placed roots, where ethylene treatment induced actin cytoskeleton disassembly within $30 \mathrm{~min}$ (diffuse signal at Fig. 2f), under gravistimulation the degradation of actin cables, especially of axial orientation, was delayed for $30 \mathrm{~min}$ (Fig. 2v).

To summarize, the treatment of $A$. thaliana seedlings with ethylene induced the disassembly of actin microfilaments in the root transition zone, which was more pronounced under vertical growth conditions, and rendered a broader spectrum of microfilament directions under gravistimulation. We hypothesize that ethylene-induced actin cytoskeleton rearrangements could underlie the "ethylene plagiotropism".

\section{Salicylic Acid Disturbes the (re)organization of Actin Microfilaments in Cortical Cells of the Arabidopsis Root Transition Zone}

Inhibitors of ethylene biosynthesis are effective to study ethylene effects on plant physiological processes. AVG, aminooxyacetic acid or rhizobitoxin are among such chemicals [14], as well as salicylic acidan endogenous non-competitive ethylene synthesis inhibitor [18, 19]. Leslie and Romani showed [18] that salicylate was able to regulate the synthesis of ethylene in plant tissues and in concentrations from 1 to $100 \mu \mathrm{M}$ effectively inhibited ethylene biosynthesis at the step of 1-aminocyclopropane-1-carboxylic acid (ACC) oxidation. In the present study we have applied $10 \mu \mathrm{M}$ salicylic acid at $\mathrm{pH}$ 6.0.

Figure 2 shows that salicylic acid disturbed the normal organization of the actin cytoskeleton in root transition zones of $A$. thaliana GFP-fABD2 seedlings. This effect appeared to be short-term in vertical growth conditions (Figs. 2c, 2g and 2k), but under gravistimulation turned to be prolonged for at least $60 \mathrm{~min}$ (Figs. 2o, 2s and 2w).

Axially aligned actin microfilaments partially disassembled in vertically oriented Arabidopsis seedlings under 30 min treatment with salicylic acid (Figs. 2c and $2 \mathrm{~g}$ ). The microfilament angle distribution spectrum (Fig. 3d) was noticeably wider than in control (Fig. 3b). Simultaneously, fractions of oblique and transversally oriented microfilaments were increased by 25 and $120 \%$, respectively (Fig. 5c). After $60 \mathrm{~min}$ of treatment with salicylic acid, the ratio between axial, oblique and transverse microfilaments returned to the control level (Figs. 2i, 2k; 5c).

During gravistimulation, salicylic acid blocked the actin cytoskeleton rearrangement (Figs. 2o, 2s and 2w), however the microfilament angle distribution spectrum was wider than in control (Figs. $3 \mathrm{~m}$ and $3 \mathrm{q}$ ). The microfilament angle distribution under gravistimulation (Fig. 5d) did not differ from the control (Fig. 4), where axially aligned microfilaments were predominant both after $30 \mathrm{~min}$ and $60 \mathrm{~min}$ (Fig. 5d).

To summarize, the treatment with salicylic acid prevented actin cytoskeleton reorganization induced by gravistimulation, and transiently disturbed axially aligned microfilaments under vertical growth conditions.

Matousková et al. [21] studied the effect of salicylic acid $(1 \mathrm{mM})$ on actin microfilament length distribution in leaf epidermal cells of $A$. thaliana. The authors showed that changes in actin cytoskeleton organization in the leaf epidermis caused by the treatment with exogenous salisylic acid remind responses triggered by 

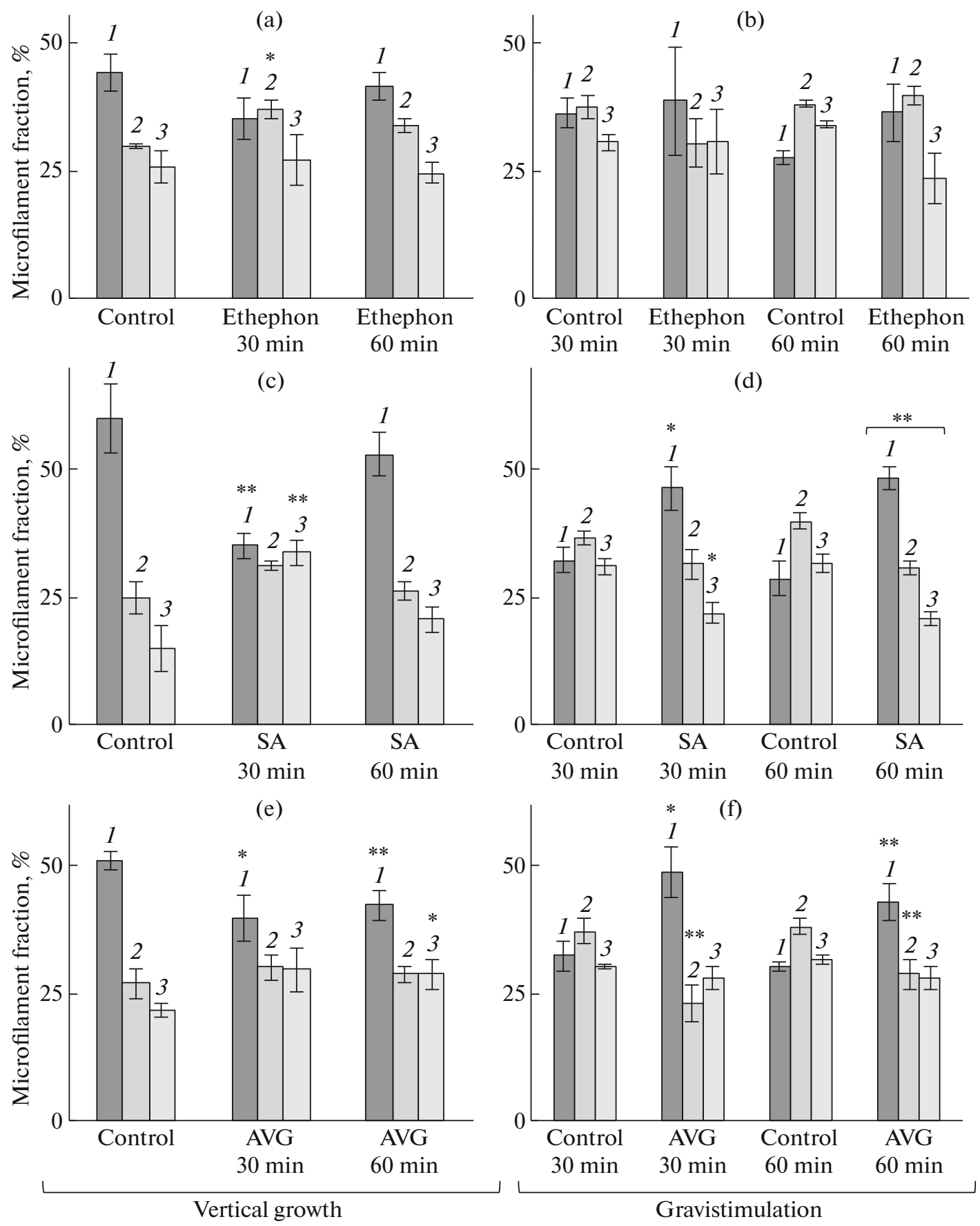

Fig. 5. Classes of microfilaments oriented axially (1), obliquely (2) or transversally (3) with respect to the root apical-basal axis in cortical cells of distal elongation zones of 6-day-old $A$. thaliana GFP- $f A B D 2$ roots in vertical growth conditions (a, c, e) or after gravistimulation (b, d, f) during 30-60 min treatment with Tris/Mes buffer ( $2 \mathrm{mM} / 4 \mathrm{mM}, \mathrm{pH} 6.0)$ containing $10 \mu \mathrm{M}$ ethephon (a, b), salicylic acid (SA) (c, d) or aminoethoxyvinylglycine (AVG) (e, f). Asterisks show significance of differences with control vertically oriented plants (Student's $t$-test): ${ }^{*} 0.01<P<0.05$; ${ }^{*} P<0.01$. Bars show the standard error.

a pathogen attack. It is interesting to note that the treatment with phosphatidic acid prevented this salycilate-induced actin rearrangement in the epidermis. In addition, treatment with actin depolymerization agents resulted in activation of expression of genes induced by salicylate, but not by jasmonate. The number of actin microfilaments in the leaf epidermis cells dramatically decreased within 10 min of treatment with salicylate, and continued to decrease in the next $20 \mathrm{~min}$. If the initial effect was minor, then microfilament number inevitably decreased after $40 \mathrm{~min}$ of treatment. Besides that, Matousková et al. [21] observed the fragmentation of actin cytoskeleton, especially of thick actin bundles, under salicylate 
treatment. After 60 min of salicylic acid treatment the number of short actin microfilaments was increased by $20 \%$. We have obtained similar results: 30 min treatment with salicylate disturbed the normal actin microfilament organization in root transition zone cells, whereas after $60 \mathrm{~min}$ of treatment the actin cytoskeleton structure reverted to the initial (Fig. 5c). However, in contrast to Matousková et al. [21], we have not found any significant changes in microfilament length under salicylic acid treatment (and AVG): microfilament length was $3.59 \pm 0.02 \mu \mathrm{m}$ under vertical growth and $3.57 \pm 0.03 \mu \mathrm{m}$ under gravistimulation.

Salicylic acid prevented the effect of actin cytoskeleton rearrangement induced by gravistimulation in root cortical cells. This mechanism could be explained by its inhibitory action on the synthesis of ethylene $[18,20]$, which is released during a stress response. We have shown earlier that salicylic acid inhibited root and shoot gravitropism of corn seedlings [19], while the treatment with ethrel (active compound-ethephon) restored gravitropism inhibited by salicylate. These results demonstrated that ethylene was essential for the normal development of a gravitropic response in corn roots and coleoptiles.

It is necessary to account for various side activities of salicylic acid in addition to ethylene synthesis inhibition. Salicylate is involved in plant stress responses, regulation of vegetative organ growth, seed germination, photosynthesis, respiration, thermogenesis, flower and seed development, senescence and programmed cell death [22]. The elevation of salicylate content in tissues led to local phytoalexin synthesis, pathogen development arrest and cell wall rigidification [23]. Hence, it is still an open question whether the actin cytoskeleton rearrangement under salicylate treatment is the consequence of ethylene synthesis inhibition, or whether it is caused by salicylate direct action, or a combination of both. Therefore, in next experiments we used a more specific inhibitor of the ACC-synthase-AVG, a derivative of vinylglycine.

\section{Aminoethoxyvinylglycine Blocks Actin Cytoskeleton Rearrangement Induced by Gravistimulation in the Root Transition Zone}

Aminoethoxyvinylglycine (AVG) is used to study the role of ethylene in plant physiology in the majority of studies [16, 24, 25]. AVG specifically blocks the synthesis of the ethylene precursor ACC from S-adenosylmethionine by the ACC synthase enzyme [26].

In our study the inhibition of ethylene synthesis by AVG in vertical growth conditions caused a fast increase in the number of actin microfilaments in the root transition zone (Fig. 2d) and expansion of microfilament orientation spectrum (Fig. 3e) within $30 \mathrm{~min}$. Axially oriented microfilaments were still predominant, however their fraction decreased by $10 \%$ (Fig. 5e). This type of actin angle distribution retained by 60 min of treatment (Figs. 3i and 5e), followed by an increase of fluorescence (Fig. 21), which suggests higher actin polymerization.

AVG clearly blocked actin cytoskeleton rearrangement induced by gravistimulation, which was an unexpected result. AVG treatment (Figs. 2p and 2t) after plant rotation by $90^{\circ}$ in the vertical plane not only increased the number of actin microfilaments and their brightness, but also the type of their angle distribution was indistinguishable from the control under vertical growth (Figs. 3n and 3r). This type of actin cytoskeleton organization was observed both after 30 and $60 \mathrm{~min}$ of gravistimulation in the presence of AVG, while the fraction of axially oriented microfilaments decreased (Fig. 5f).

Our experiments show that action of AVG as a blocker of actin rearrangement under gravistimulation is notably stronger than the effect of salicylic acid in the same concentration $(10 \mu \mathrm{M})$ (Figs. $2 \mathrm{~g}, 2 \mathrm{~h}, 2 \mathrm{k}, 2 \mathrm{l}, 2 \mathrm{~s}, 2 \mathrm{t}$, $2 \mathrm{w}$ and $2 \mathrm{x}$ ). On the other hand, exogenous ethylene stimulated the disassembly of actin microfilaments and maximally (in comparison with other treatments) broadened the actin angle distribution spectrum (Figs. 2f, 2j, 2r, 2v and 2c, 2g, 2l, 2p). These data support that ethylene synthesis is essential for gravityinduced actin cytoskeleton rearrangement in Arabidopsis root cells.

The mechanism of ethylene effect on the development of the gravity response is not exactly known yet. Several studies reported that ethylene does not play a significant role in gravitropism and in low amount only modulates growth, or acts as a mediator in auxin and brassinosteroid pathways, thus enhancing the gravitropic response [24, 27]. Later on Muday and colleagues [28] used Arabidopsis mutants on ethylene reception and signaling (ethylene resistant $1-1$ (etr 1-1) and ethylene insensitive2-1 (ein2-1)) and found evidence for two independent pathways of ethylene involvement into regulation of gravitropism: 1) suppression of gravitropic response in ein2-1 mutants, and 2) wild type-like development of gravitropic response in etr1-1. The other study reports that ACC synthase of Arabidopsis ACS7 is involved into gravitropism and may even serve as the point of cross-talk with calcium signaling since it is the target for calcium-dependent protein kinases [29].

The first step of the gravity response in roots is gravity stimulus perception, initiated by statoliths in columella cells. The involvement of the distal elongation zone, the so-called transition zone, is also required in the next step of gravity response in the root (see $[30,31]$ ). The gravitropic bending is the result of differential growth in upper and lower parts of this region. The distal elongation zone was also shown to be the most active for vesicular transport and polar auxin transport in basipetal direction, mediated by PIN2 [32]. Under treatment of Arabidopsis seedlings with brefeldin A, an inhibitor of auxin polar transport, brefeldin-induced compartments were most abundant in the distal elongation zone as well $[6,31]$. It was also 
Aminoethoxyvinylglycine (AVG, $10 \mu \mathrm{M}$ ) effect on root length increase (mm) of $A$. thaliana seedlings

\begin{tabular}{l|c|c|c|c}
\hline \multirow{2}{*}{ Experiment } & \multicolumn{4}{|c}{ Time, min } \\
\cline { 2 - 4 } Vertical growth: & 30 & 60 & 180 & 360 \\
Control & $0.10 \pm 0.01$ & $0.23 \pm 0.01$ & $1.13 \pm 0.04$ & $2.64 \pm 0.08$ \\
AVG & $0.14 \pm 0.01^{*}$ & $0.39 \pm 0.02^{* *}$ & $1.71 \pm 0.07^{* *}$ & $3.76 \pm 0.16^{* *}$ \\
Gravistimulation: & $0.03 \pm 0.01$ & $0.19 \pm 0.02$ & $1.13 \pm 0.06$ & $2.71 \pm 0.12$ \\
Control & $0.16 \pm 0.04^{* *}$ & $0.33 \pm 0.03^{* *}$ & $1.35 \pm 0.05^{*}$ & $3.08 \pm 0.11^{*}$ \\
AVG
\end{tabular}

The numbers shown are root length increase \pm standard error. Asterisks show the significance of differences in root length increase in control and after treatment with AVG (at least 20 seedling in three replicates, Student's $t$-test): $* 0.01<P<0.05 ; * * P<0.01$

shown that in this region ethylene induced reactive oxygen species in the apoplast, higher callose deposition in the cell wall, and reduced cell elongation [33]. In contrast, cell length in the distal elongation zone was increased in seedlings treated with AVG. We suppose that this explains the increased root growth rate in Arabidopsis after AVG treatment by $42 \%$ in vertical position and by $14 \%$ under gravistimulation (table). It could be assumed that ethylene is related to differential growth of gravistimulated roots by regulating cell wall polysaccharide composition, but due to the fast diffusion, its concentration must equalize shortly within Arabidopsis root. Actin polymerization/depolymerization regulators or ARP2/3 actin-binding proteins which direct microfilament branching [34] could serve as other potential targets for ethylene action.

In our experiments an ethylene synthesis block led to the stabilization of microfilaments in the axial direction, whereas ethylene treatment, in contrast, induced a "randomization" of the actin cytoskeleton (Figs. 2f, 2j, $2 \mathrm{r}, 2 \mathrm{v} ; 3 \mathrm{c}, 3 \mathrm{~g}, 3 \mathrm{l}, 3 \mathrm{p}$ ). This phenomenon is similar to deep reorganization of microtubules in the root elongation zone of corn after ethylene treatment, reported by Baluška et al. [31, 35], however, microtubule rearrangement was not correlated with growth rate.

Actin "randomization", however, could potentially have a more important physiological value. It is known that in cells leaving the root apical meristem, a fast switch of growth type from "isodiametric" to extension growth corresponds to the transition of actin cytoskeleton organization from perinuclear to axial [31]. Dhonuksche et al. [6] showed, using plants and yeast and mammalian cell models, that inhibitors of auxin polar transport-brefeldin A, 2,3,5-triiodbenzoic acid and 2-(1-pyrenoyl)benzoic acid-disrupted vesicular transport and PIN traffic in plants, inhibited auxin efflux from plant cells and stabilized the actin cytoskeleton. However, inhibitors of auxin polar transport were not shown to affect actin polymerization/depolymerization directly [6]. To conclude, lateral PIN distribution appears to be necessary for lateral redistribution of PINs in columella cells and the transition zone, and subsequent reorientation of polar auxin flux to lateral direction-parallel to the gravity vector along transversally oriented microfilaments. In contrast, the stabilization of axial elements of the cytoskeleton (i.e. restricted spectrum of microfilament orientations under treatment with AVG) may reduce the ability of the root transition zone to develop the response to gravity.

To conclude, we propose that the role of ethylene in the regulation of the root gravitropic response is to randomize actin microfilament orientation, thus enhancing the probability of auxin polar transport in lateral direction.

\section{ACKNOWLEDGMENTS}

The authors acknowledge Saint Petersburg State University for research grants nos. 1.38.233.2014, 1.42.1288.2014, 1.57.1157.2014, 1.57.163.2015, and the Russian Foundation for Basic Research, project no. 14-04-01624a. Confocal microscopy experiments were carried out with support of Research Resource Center "Molecular and Cell Technologies" of St. Petersburg State University, project nos. 109-72 and 109-244. K. Vissenberg acknowledges the University of Antwerp and the Research Foundation Flanders, project nos. G009412N, G.0.602.11.N.10 and 1.5.091.11.N.00.

\section{REFERENCES}

1. Sato, E.M., Hijazi, H., M.J. Bennett, M.J., Vissenberg, K. and Swarup, R., New insights into the dynamics of root gravitropic signalling, J. Exp. Bot., 2015, vol. 66, p. 2155-2165.

2. Medvedev, S.S., Mechanisms and physiological role of polarity in plants, Russ. J. Plant Physiol., 2012, vol. 59, pp. 502-514.

3. Neljubow, D., Über die horizontale Nutation der Stengel von Pisum sativum und einiger anderer Pflanzen, Beih. Bot. Centralbl., 1901, vol. 10, pp. 128-138.

4. Morita, M.T., Directional gravity sensing in gravitropism, Annu. Rev. Plant Biol., 2010, vol. 61, pp. 705-720.

5. Blancaflor, E.B., Regulation of plant gravity sensing and signaling by the actin cytoskeleton, Am. J. Bot., 2013, vol. 100, pp. 143-152.

6. Dhonukshe, P., Tanaka, H., Goh, T., Ebine, K., Mähönen, A., Prasad, K., Blilou, I., Geldner, N., Xu, J., Uemura, T., Chory, J., Ueda, T., Nakano, A., Scheres, B., and Friml, J., Generation of cell polarity 
in plants links endocytosis, auxin distribution and cell fate decisions, Nature, 2008, vol. 456, pp. 962-966.

7. Rahman, A., Takahashi, M., Shibasaki, K., Wu, S., Inaba, T., Tsurumi, S., and Baskin, T.I., Gravitropism of Arabidopsis thaliana roots requires the polarization of PIN2 toward the root tip in meristematic cortical cells, Plant Cell, 2010, vol. 22, pp. 1762-1776.

8. Pozhvanov, G.A., Suslov, D.V., and Medvedev, S.S., Actin cytoskeleton rearrangements during the gravitropic response of Arabidopsis roots, Tsitologiya, 2013, vol. 55 , pp. $28-35$.

9. Nick, P. Han, M.-J., and An, G., Auxin stimulates its own transport by shaping actin filaments, Plant Physiol., 2009, vol. 151, pp. 155-167.

10. Zhao, Y., Zhao, S., Mao, T., Qu, X., Cao, W., Zhang, L., Zhang, W., He, L., Li, S., Ren, S., Zhao, J., Zhu, G., Huang, S., Ye, K., Ming, Y.M., et al., The plant-specific actin binding protein SCAB1 stabilizes actin filaments and regulates stomatal movement in Arabidopsis, Plant Cell, 2011, vol. 23, pp. 2314-2330.

11. Sheahan, M.B., Staiger, C.J., Rose, R.J., and McCurdy, D.W., A green fluorescent protein fusion to actin-binding domain 2 of Arabidopsis fimbrin highlights new features of a dynamic actin cytoskeleton in live plant cells, Plant Physiol., 2004, vol. 136, pp. 39683978.

12. Jacques, E., Buytaert, J., Wells, D.M., Lewandowski, M., Bennett, M.J., Dirckx, J., Verbelen, J.P., and Vissenberg, K., MicroFilament Analyzer, an image analysis tool for quantifying fibrillar orientation, reveals changes in microtubule organization during gravitropism, Plant J., 2013, vol. 74, pp. $1045-1058$.

13. Baluška, F., Barlow, P.W., Baskin, T.I., Chen, R., Feldman, L., Forde, B.G., Geisler, M., Jernstedt, J., Menzel, D., Muday, G.K., and Murphy, A., What is apical and what is basal in plant root development? Trends Plant Sci., 2005, vol. 10, pp. 409-411.

14. Wheeler, R.M. and Salisbury, F.B., Gravitropism in higher plant shoots. I. A role for ethylene, Plant Physiol., 1981, vol. 67, pp. 686-690.

15. Kramer, S., Piotrowski, M., Èhnemann, F.K., and Edelmann, H.G., Physiological and biochemical characterization of ethylene-generated gravicompetence in primary shoots of coleoptile-less gravi-incompetent rye seedlings, J. Exp. Bot., 2003, vol. 54, pp. 2723-2732.

16. Edelmann, H.G. and Roth, U., Gravitropic plant growth regulation and ethylene: an unsought cardinal coordinate for a disused model, Protoplasma, 2006, vol. 229, pp. 183-191.

17. Harrison, M.A. and Pickard, B.G., Evaluation of ethylene as a mediator of gravitropism by tomato hypocotyls, Plant Physiol., 1986, vol. 80, pp. 592-595.

18. Leslie, C.A. and Romani, R.J., Inhibition of ethylene biosynthesis by salicylic acid, Plant Physiol., 1988, vol. 88, pp. 833-837.

19. Medvedev, S.S. and Markova, I.V., Participation of salicylic acid in plant gravitropism, Dokl. Akad. Nauk SSSR, 1991, vol. 316, pp. 1014-1016.

20. Lee, J.H., Jin, E.S., and Kim, W.T., Inhibition of auxin-induced ethylene production by salicylic acid in mung bean hypocotyls, J. Plant Biol., 1999, vol. 42, pp. $1-7$.

21. Matoušková, J., Janda, M., Fišer, R., Šašek, V., Kocourková, D., Burketová, L., Dušková, J., Mar- tinec, J., and Valentová, O., Changes in actin dynamics are involved in salicylic acid signaling pathway, Plant Sci., 2014, vol. 223, pp. 36-44.

22. Vicente, M.R. and Plasencia, J., Salicylic acid beyond defence: its role in plant growth and development, J. Exp. Bot., 2011, vol. 62, pp. 3321-3338.

23. Durango, D., Pulgarin, N., Echeverri, F., Escobar, G., and Quiñones, W., Effect of salicylic acid and structurally related compounds in the accumulation of phytoalexins in cotyledons of common bean (Phaseolus vulgaris L.) cultivars, Molecules, 2013, vol. 9, pp. 10 60910628 .

24. Madlung, A., Behringer, F.J., and Lomax, T.L., Ethylene plays multiple nonprimary roles in modulating the gravitropic response in tomato, Plant Physiol., 1999, vol. 120, pp. 897-906.

25. Gupta, A., Singh, M., Jones, A.M., and Laxmi, A., Hypocotyl directional growth in Arabidopsis: a complex trait, Plant Physiol., 2012, vol. 159, pp. 1463-1476.

26. Yu, Y.B. and Yang, S.F., Auxin-induced ethylene production and its inhibition by aminoethoxyvinyiglycine and cobalt ion, Plant Physiol., 1979, vol. 64, pp. 10741077.

27. Chang, S.C., Kim, Y.S.K., Lee, J.Y., Kaufman, P.B., Kirakosyan, A., Yun, H.S., Kim, T.W., Kim, S.Y., Cho, M.H., Lee, J.S., and Kim, S.K., Brassinolide interacts with auxin and ethylene in the root gravitropic response of maize (Zea mays), Physiol. Plant., 2004, vol. 121, pp. 666-673.

28. Muday, G.K., Brady, S.R., Argueso, C., Deruère, J., Kieber, J.J., and DeLong, A., RCN1-regulated phosphatase activity and EIN2 modulate hypocotyl gravitropism by a mechanism that does not require ethylene signaling, Plant Physiol., 2006, vol. 141, pp. 1617-1629.

29. Huang, S.J., Chang, C.L., Wang, P.H., Tsai, M.C., Hsu, P.H., and Chang, F., A type III ACC synthase, ACS7, is involved in root gravitropism in Arabidopsis thaliana, J. Exp. Bot., 2013, vol. 64, pp. 4343-4360.

30. Verbelen, J.P., Cnodder, T.D., Le, J., Vissenberg, K., and Baluška, F., The root apex of Arabidopsis thaliana consists of four distinct zones of growth activities: meristematic zone, transition zone, fast elongation zone and growth terminating zone, Plant Signal. Behav., 2006, vol. 1, pp. 296-304.

31. Baluška, F., Mancuso, S., Volkmann, D., and Barlow, P.W., Root apex transition zone: a signallingresponse nexus in the root, Trends Plant Sci., 2010, vol. 15, pp. 402-408.

32. Friml, J., Wiśniewska, J., Benková, E., Mendgen, K., and Palme, K., Lateral relocation of auxin efflux regulator PIN3 mediates tropism in Arabidopsis, Nature, 2002, vol. 415, pp. 806-809.

33. De Cnodder, T., Vissenberg, K., van der Straeten, D., and Verbelen, J.P., Regulation of cell length in the Arabidopsis thaliana root by the ethylene precursor 1-aminocyclopropane-1-carboxylic acid: a matter of apoplastic reactions, New Phytol., 2005, vol. 168, pp. 541-550.

34. Kandasamy, M.K., Deal, R.B., McKinney, E.C., and Meagher, R.B., Plant actin-related proteins, Trends Plant Sci., 2004, vol. 9, pp. 196-202.

35. Baluška, F., Volkmann, D., and Barlow, B.W., A polarity crossroad in the transition growth zone of maize root apices: cytoskeletal and developmental implications, J. Plant Growth Regul., 2001, vol. 20, pp. 170-181. 